\title{
Identification of causative pathogen and its antibiotic sensitivity in cases of preterm premature rupture of membranes
}

\author{
Sai Prasanna Kandukuri*, Ramamani Chadalawada, Bhavishya Gollapalli
}

Department of Obstetrics and Gynecology, Narayana Medical College and Hospital, Nellore, Andhra Pradesh, India

Received: 14 September 2019

Accepted: 30 September 2019

\section{*Correspondence:}

Dr. Sai Prasanna Kandukuri,

E-mail: kandkurisaiprasanna@gmail.com

Copyright: (C) the author(s), publisher and licensee Medip Academy. This is an open-access article distributed under the terms of the Creative Commons Attribution Non-Commercial License, which permits unrestricted non-commercial use, distribution, and reproduction in any medium, provided the original work is properly cited.

\begin{abstract}
Background: Pre-labor rupture of membranes is defined as amniotic membrane rupture before the onset of labor contractions, and if it happens before 37 weeks, it is called preterm premature rupture of membranes (PPROM). Several organisms commonly present in the vaginal tract are E.coli, Group-B streptococci, staphylococcus aureus, chlamydia trachomatis, Gardnerella vaginalis and Enterococcus faecalis which secrete proteases that degrade collagen thereby weakening the fetal membranes leading to PPROM. Appropriate antibiotic therapy has a significant role in the prevention and treatment of maternal and neonatal complications.

Methods: This was a prospective observational study done in the department of obstetrics and gynaecology, Narayana medical college, Nellore. Selectively 100 patients with complaint of PPROM admitted to labor room were included in the study. Diagnosis of membrane rupture was established by speculum examination, and high vaginal swabs are taken and sent to laboratory for identifying bacteria using gram staining and cultured in aerobic and anaerobic methods. Antimicrobial susceptibility testing of the organisms was performed by disk diffusion method by Kirby and Bauer.

Results: Out of 100, high vaginal swabs had growth in 82 patients, and 18 were sterile. The repeatedly isolated organism in patients with PPROM is E.coli amounting 32\%, followed by candidal species $20 \%$. Staphylococci are scoring $11 \%$ and enterococci $8 \%$. However, organisms like gardenella vaginalis and Group B streptococcus are least common with a score of $6 \%$ and $5 \%$ respectively. In this study, E.coli is highly sensitive to tigecycline, colistin $100 \%$ each and highly resistant to gentamycin and amikacin.

Conclusions: In this study, E.coli is related to the maximum number of cases with preterm premature rupture of membranes. Appropriate use of antibiotics significantly lowers maternal morbidity and neonatal mortality.
\end{abstract}

Keywords: Antibiotics, High vaginal swab, Preterm premature rupture of membranes, Premature rupture of membranes

\section{INTRODUCTION}

Pre-labor rupture of membranes previously known as preterm rupture of membranes is described as amniotic membrane rupture before the onset of labor contractions, and if it happens before 37 weeks, it is called preterm premature rupture of membranes (PPROM). ${ }^{1}$ Preterm labor is one of the major causes of perinatal mortality and neurological morbidity. ${ }^{2}$ Imbalance in vaginal bacterial colonization in pregnancy makes these women susceptible to the colonization of pathogenic organisms. ${ }^{3}$ Among women with preterm PROM, clinically evident intraamniotic infection occurs in approximately $15-25 \%$ and postpartum infection occurs in approximately 1520\%. Collagens are the structural component for the tensile strength of membranes. The foremost cause for rupture of membranes is loss of tensile power of amniotic membranes. The pathophysiology of infection-causing 
PPROM is considered to be due to the production of prostaglandins and matrix-degrading enzymes via microbial endotoxins and pro-inflammatory cytokines (IL-8, IL 1beta, TNF alpha) which are liberated after binding of organisms to pattern- recognition receptors (ex-toll like receptors). ${ }^{5}$ This lead to an increase in metalloproteinase, which is responsible for collagen degradation leading to a decrease in the tensile power of membranes resulting in rupture. The hypothesis is that several organisms that are commonly present in the vaginal flora, including E.coli, $B$ streptococci, staphylococcus aureus and microorganisms that cause bacterial vaginosis, secrete proteases that degrade collagen and weaken the fetal membranes leading to PROM. ${ }^{6,7}$ With regard to the importance of maternal genital tract bacterial colonization as an etiologic factor in PPROM, appropriate antibiotic therapy has a cardinal role in the prevention and treatment of maternal and neonatal complications.

The objective of this study was to diagnose the appropriate organism using a high vaginal swab. To select an appropriate antibiotic therapy to prevent maternal and neonatal complications.

\section{METHODS}

This study was prospective observational study from October 2018 to April 2019. Data of 100 patients with singleton pregnancy are admitted to the labor room with complaint of PPROM. Detailed history regarding gestational age, parity, duration of the leak was collected. In detail, the general and obstetrical examination is performed. Diagnosis of membrane rupture was accomplished by proper speculum examination to see amniotic fluid leakage from the cervical os. The amount, color, and smell of the fluid were assessed. Specimens were gram stained and cultured in both aerobic and anaerobic methods for identification of pathogens. In aerobic culture, bacterial colonies are identified by their morphology and biochemical reactions like catalase, coagulase, and esculin hydrolysis for gram-positive organisms. In the case of gram-negative bacteria, testing for motility, catalase, oxidase, sugar fermentation, nitrate reduction, citrate utilization, urea hydrolysis, methyl red, and Voges-Proskauer test is done. In anaerobic culture, bacterial identification was done by colony morphology, pitting of agar, growth character in selected and indicator anaerobic media plates. Presence of candida species in gram stained preparation of vaginal discharge showed gram positive budding yeast cells.

\section{Antimicrobial susceptibility testing}

It is done using a disc diffusion method of KIRBY and BAUER. ${ }^{8}$ The test organism is inoculated into protease peptone water and incubated for 4-6 hours. The turbidity is matched with McFarland opacity standard 0.5. A sterile cotton swab is immersed into the suspension is used to streak the surface of the Mueller-Hinton agar. Different antimicrobial discs, $6.0 \mathrm{~mm}$ in diameter, charged with appropriate concentrations of antibiotic are placed at a distance of $20 \mathrm{~mm}$ apart; inverted plates are incubated for 16-24 hours at $37^{\circ} \mathrm{C}$. The degree of antibiotic sensitivity is obtained by measuring the zones of inhibition of growth around the disks.

- Susceptible: zone of inhibition of $>10 \mathrm{~mm}$

- Resistant: zone of inhibition $<10 \mathrm{~mm}$.

\section{Inclusion criteria}

- $\quad$ Singleton pregnancy

- PPROM

- Gestational age between 24-37 weeks

- Chorioamnionitis

- No prior antibiotic use.

\section{Exclusion criteria}

- Multiple gestations

- Patients consuming alcohol and smoking tobacco

- Patients refusal for being in the study.

\section{RESULTS}

A total of 100 cases with PPROM are taken a high vaginal swab. Out of 100 cases of vaginal swab culture study, 82 cases were culture positive, and 18 cases were sterile (Figure 1).

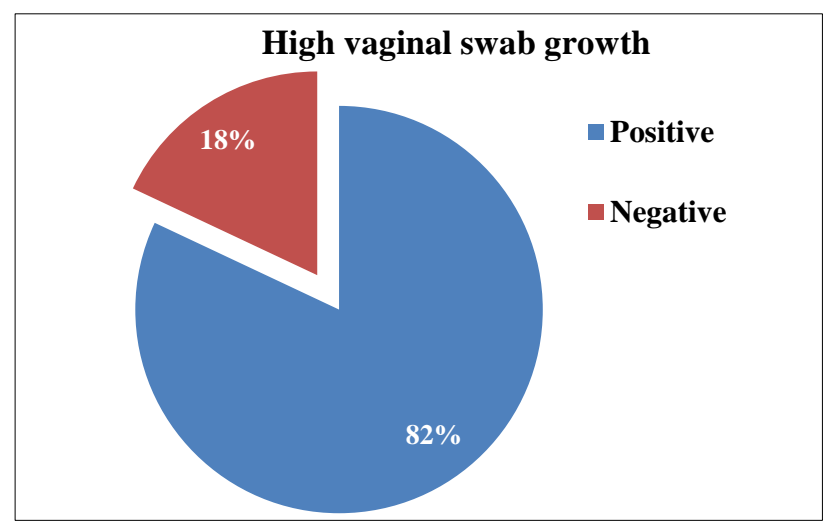

Figure 1: Baseline characteristics of total study population.

Table 1: Organisms associated with PPROM.

\begin{tabular}{|c|c|}
\hline Organism & Total percentage \\
\hline Escherichia coli & $32 \%$ \\
\hline Candida & $20 \%$ \\
\hline Staphylococci & $11 \%$ \\
\hline Enterococci & $8 \%$ \\
\hline Gardnerella vaginalis & $6 \%$ \\
\hline Group B streptococcus & $5 \%$ \\
\hline No growth & $18 \%$ \\
\hline
\end{tabular}


The most common isolated organism is E.coli among all cases amounting $32 \%$, followed by candida species $20 \%$, staphylococci scoring $11 \%$ and enterococci $8 \%$.
However, pathogens like Gardnerella vaginalis and Group B streptococcus are least common with a score of $6 \%$ and $5 \%$ respectively (Table 1 ).

Table 2: Antibiotic susceptibility of following organisms.

\begin{tabular}{|c|c|c|c|c|c|c|}
\hline Sr. no. & Antibiotics & E.coli & Staphylococci & Enterococci & G. vaginalis & Group B streptococci \\
\hline 1 & Tigecycline & $100 \%$ & $80 \%$ & $80 \%$ & N/A & N/A \\
\hline 2 & Colistin & $100 \%$ & N/A & N/A & N/A & N/A \\
\hline 3 & Linezolid & $60 \%$ & $100 \%$ & $100 \%$ & N/A & N/A \\
\hline 4 & Gentamycin & $20 \%$ & $90 \%$ & $80 \%$ & $80 \%$ & $80 \%$ \\
\hline 5 & Amikacin & $20 \%$ & N/A & $20 \%$ & N/A & $60 \%$ \\
\hline 6 & Erythromycin & N/A & $20 \%$ & N/A & N/A & $30 \%$ \\
\hline 7 & Vancomycin & N/A & $60 \%$ & $30 \%$ & N/A & N/A \\
\hline 8 & Ciprofloxacin & N/A & N/A & $80 \%$ & $60 \%$ & N/A \\
\hline 9 & Cefotaxime & $80 \%$ & N/A & N/A & N/A & $100 \%$ \\
\hline 10 & $\begin{array}{l}\text { Amoxycillin+ } \\
\text { clavulanic acid }\end{array}$ & N/A & N/A & $60 \%$ & N/A & N/A \\
\hline 11 & Nitrofurantoin & $60 \%$ & $80 \%$ & N/A & N/A & $40 \%$ \\
\hline 12 & Metronidazole & N/A & N/A & N/A & $100 \%$ & N/A \\
\hline
\end{tabular}

E.coli is highly sensitive to Tigecycline, colistin $100 \%$ each and highly resistant to Gentamycin and Amikacin. Staphylococci were more sensitive to Linezolid $100 \%$, Gentamycin 90\%, Tigecycline, and Nitrofurantoin each $80 \%$. It is mostly resistant to Vancomycin $60 \%$ and Erythromycin 20\%. Enterococcus species are highly sensitive to Linezolid 100\%, Tigecycline $80 \%$, Gentamycin, Ciprofloxacin each $80 \%$ and it is resistant to Amikacin 20\% and Vancomycin 30\% (Table 2).

\section{DISCUSSION}

In this prospective observational study, antibiotic sensitivity is determined by taking high vaginal swabs in women with PPROM. E.coli is cited as one of the most common organisms amounting $32 \%$, followed by candida $20 \%$. In the antibiogram study, it is highly sensitive to Tigecycline $100 \%$, Colistin $100 \%$, and Cefotaxime $80 \%$. It is mostly resistant to Gentamycin $20 \%$ and Amikacin $20 \%$. This study found that staphylococci were more sensitive to Linezolid 100\%, Gentamycin 90\%, Tigecycline, and Nitrofurantoin each $80 \%$. It is mostly resistant to Vancomycin $60 \%$ and Erythromycin $20 \%$. Enterococcus species are highly sensitive to Linezolid $100 \%$, Tigecycline $80 \%$, Gentamycin, Ciprofloxacin each $80 \%$ and it is resistant to Amikacin 20\% and Vancomycin $30 \%$. Then comes $G$. vaginalis $6 \%$ and eventually Group B streptococcus $5 \%$.

Administration of broad-spectrum antibiotics prolongs pregnancy, reduces maternal and neonatal infections, and reduces gestational age-dependent morbidity. ${ }^{9}$ Based on available information, in order to reduce maternal and neonatal infections and gestational-age-dependent morbidity, a 7-day course of therapy with a combination of intravenous ampicillin and erythromycin followed by oral amoxicillin and erythromycin is recommended during expectant management of women with PROM. ${ }^{10}$

\section{CONCLUSION}

Proper detection of causative pathogens by high vaginal swab and administration of appropriate antibiotic therapy improves maternal and neonatal outcome. Most of the pathological isolates were sensitive to Tigecycline, Linezolid, and Gentamycin in our study.

Funding: No funding sources Conflict of interest: None declared

Ethical approval: The study was approved by the Institutional Ethics Committee

\section{REFERENCES}

1. Prelabor rupture of membranes. ACOG Practice Bulletin No. 188. American College of Obstetricians and Gynecologists. Obstet Gynecol. 2018;131:e1-14.

2. Getahun D, Ananth CV, Oyelese Y, Peltier MR, Smulian JC, Vintzileos AM. Acute and chronic respiratory diseases in pregnancy: associations with spontaneous premature rupture of membranes. J Maternal Fetal Neonatal Med. 2007;20:669-75.

3. Lajos GJ, Passini Junior R, Nomura ML, Amaral E, Pereira BG, Milanez $\mathrm{H}$, et al. Cervical bacterial colonization in women with preterm labor or premature rupture of membranes. Rev Bras Ginecol Obstet. 2008;30:393-9. 
4. Kenyon S, Boulvain M, Neilson JP. Antibiotics for preterm rupture of membranes. Cochrane Database Systematic Reviews. 2010;8:CD001058.

5. Goldenberg RL, Culhane JF, Iams JD. Epidemiology and causes of preterm birth. Lancet. 2008;371:75-84.

6. McGregor JA, French JI, Lawellin D. Bacterial protease-induced reduction of chorioamniotic membrane strength and elasticity. Obstet Gynecol. 1987;69:167-74.

7. Draper D, Jones W, Heine RP. Trichomonas vaginalis weakens human amniochorion in an in vitro model of premature membrane rupture. Infect Dis Obstet Gynecol. 1995;2:267-74.

8. Jorgensen JH, Turnidge JD. Murray PR, Baron EJ, Landry ML, Pfaller MA. Antibacterial susceptibility tests: dilution and disk diffusion methods, Manual of clinical microbiology, $9^{\text {th }}$ ed. Washington, DC. Am Society Microbiol; 2007:1152-1172.
9. Kenyon S, Boulvain M, Neilson JP. Antibiotics for preterm rupture of membranes. Cochrane Database of Systematic Reviews. 2010;8:CD001058.

10. Kenyon SL, Taylor DJ, Tarnow-Mordi W. Broadspectrum antibiotics for preterm, prelabour rupture of fetal membranes: the ORACLE I randomised trial. ORACLE Collaborative Group. Lancet. 2001;357:979-88.

Cite this article as: Kandukuri SP, Chadalawada R, Gollapalli B. Identification of causative pathogen and its antibiotic sensitivity in cases of preterm premature rupture of membranes. Int $\mathrm{J}$ Reprod Contracept Obstet Gynecol 2019;8:4250-3. 